\title{
OPEN Fitness for purpose of stabilized stool samples for bile acid metabolite analyses
}

\author{
Lorie Neuberger-Castillo ${ }^{1 凶}$, Wim Ammerlaan ${ }^{1}$ \& Fay Betsou ${ }^{2}$
}

Biobanks and cohort studies are increasingly utilizing chemical stabilizers to collect and store stool samples for downstream DNA-based microbiome analyses. While stabilizers permit ambienttemperature collection and storage of samples for gut microbiome studies, the use of the same sample type for downstream metabolomics assays has not been explored. Microbiome-metabolomics analysis of fecal samples is increasingly getting attention to further elucidate the mechanisms by which the gut microbiota influences the host. In this study, we evaluated fitness-for-purpose of OMNIgene-GUT-collected stool samples for downstream metabolomics assays in the scope of fecal bile acids (BA) quantification. Biocrates Bile Acids Kit was used for the quantification of BA from eight healthy donors' samples collected in (1) OMNIgene-GUT kit and (2) snap frozen in $-80^{\circ} \mathrm{C}$ in duplicates. A highly selective reversed phase LC-MS/MS analysis method in negative ion multiple reaction monitoring (MRM) detection mode was applied to determine the BA concentrations in each sample. Total fecal BA levels were detectable in OMNIgene-GUT-collected samples (range: 29.9-903.7 pmol/ $\mathrm{mg}$ ). Paired t-test confirmed that there was a significant difference in the total BAs between the OMNIgene-GUT and snap frozen samples $(p<0.05)$. Extractions from snap frozen samples resulted in higher concentrations of total BAs (range: $243.7-1136.2 \mathrm{pmol} / \mathrm{mg}$ ). Qualitative differences between individual donors' BA profiles were detectable using the two sample collection methods. No significant difference was found in the relative concentrations of primary (CA, CDCA) or secondary (DCA, LCA, UDCA) unconjugated BAs to the total BA concentrations in OMNIgene-GUT-collected samples as compared with the snap frozen samples (Wilcoxon-Mann-Whitney test, $p>0.05$ ). PassingBablok method comparison and correlation analyis showed a high degree of correlation in the relative concentrations of CA, CDCA, DCA and LCA between OMNIgene-GUT and snap frozen samples. For these four bile acids, the two methods are comparable at an acceptability bias of $30 \%$. We conclude that the OMNIgene-GUT-collected stool samples are fit-for-purpose for downstream fecal bile acids analysis.

Previous studies have established a bidirectional relationship between the host bile acid homeostasis and the gut microbiota, and bacterial overgrowth has been associated with intestinal inflammation and reduced bile acid concentrations in the gut ${ }^{1-3}$. Bile acids (BAs) are natural products of cholesterol synthesis that aid in the emulsification and absorption of dietary fats in the small intestine and are considered to be as important as hormones ${ }^{4}$. The primary BAs, cholic acid (CA) and chenodeoxycholic acid (CDCA), are synthesized in the liver and largely conjugated with glycine and taurine. After performing their critically important function of promoting intestinal fat absorption, approximately $95 \%$ are absorbed by ileal BA transporters for recycling back to the liver. The remaining $5 \%$ pass to the colon where most undergo other microbiota-mediated biotransformations (e.g. 7-alpha-dehydroxylation from primary to secondary bile acids). Only a small amount of secondary bile acids are reabsorbed by passive diffusion, most is excreted in faeces ${ }^{5}$.

The gut microbiota can change the amount and composition of the BA pool through their effects on BA metabolism, specifically in synthesis, deconjugation and conversion of primary to secondary BA ${ }^{1}$. The quantification of fecal bile acids and the profiling of the gut microbial community are increasingly getting attention to further elucidate the mechanisms by which the gut microbiota influences the host ${ }^{3,5}$. One study which evaluated adults with non-alcoholic fatty liver disease (NAFLD) concluded that gut microbiota dysbiosis is associated with altered bile acid (BA) homeostasis, which renders the patients at increased risk of hepatic injury ${ }^{6}$. 


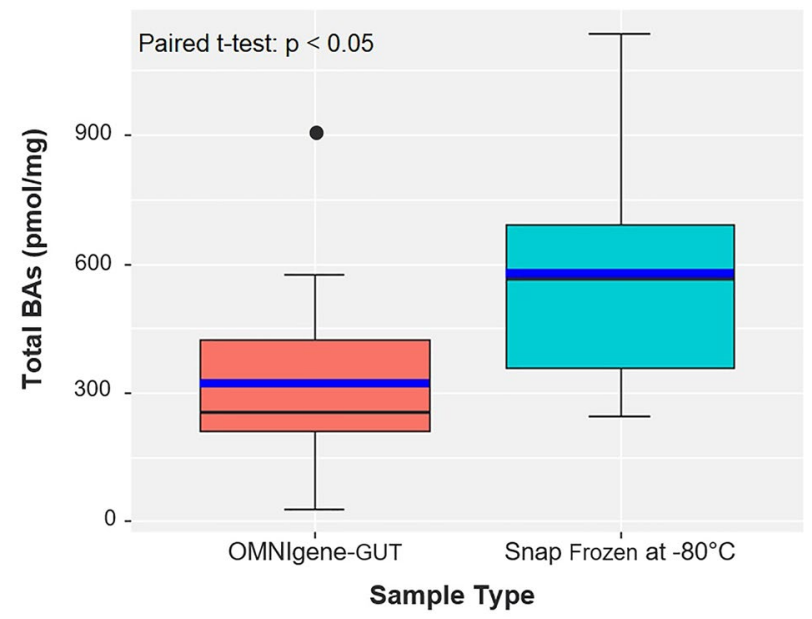

Figure 1. Total fecal bile acid concentrations (pmol) per mg stool, measured from human stool samples stabilized in OMNIgene-GUT and Snap frozen at $-80^{\circ} \mathrm{C}$. The box/whiskers represent the following: 1st Quartile, Median, 3rd Quartile. The blue line represents the Mean. The error bars represent the standard deviation. Paired t-test, $\mathrm{p}=0.0029$.

To be able to make meaningful statements about the correlation between the BA homeostasis and gut microbiota composition, it is essential that the same stool sample be used for both analyses. The collection method, which is considered as the gold standard method for downstream analysis of metabolites and the gut microbiome composition, is the immediate freezing of stool samples ${ }^{7,8}$. Previous studies showed that freezing samples in $-20{ }^{\circ} \mathrm{C}$ or lower immediately after collection preserves the microbial composition similar to analysis of a fresh sample and also avoids potential influence of added preservative. While preserving the microbial composition, it also preserves the detectability of metabolites ${ }^{7,8}$.

However, this approach is not always feasible in large-scale, population-based studies, home collection and collection in remote areas because of the high costs associated with cold-chain shipping requirements.

On the other hand, biobanks and large-cohort studies usually collect stabilized stool samples with collection kits, such as the OMNIgene-GUT kit, which has extensively been validated for downstream DNA-based microbiome analyses ${ }^{9}$. As per the manufacturer, the mechanism of stabilization is through inhibition of microbial growth and DNA degradation ${ }^{10}$.

While such a kit permits ambient-temperature collection and storage of samples for gut microbiome studies, the use of the same sample type for downstream targeted bile acid quantitative assays has not been explored. Integrated microbiome-metabolomics analyses of fecal samples are increasingly getting attention to further elucidate the mechanisms by which the gut microbiota influences the host. Therefore in this study, we evaluated the fitness-for-purpose of the OMNIgene-GUT-collected stool samples for downstream metabolomics assays in the scope of the fecal bile acids (BA) quantification.

\section{Results}

The concentration of total fecal BA levels could be measured in OMNIgene-GUT-collected samples (mean: $323.8 \mathrm{pmol} / \mathrm{mg}$; range: $29.9-903.7 \mathrm{pmol} / \mathrm{mg}$ ) (Figs. 1, 2 and Table 1). Paired t-test confirmed that there was a significant difference in the total BAs between the OMNIgene-GUT and snap frozen samples $(\mathrm{p}<0.05)$. Extractions from snap frozen samples resulted in higher concentrations of total BAs (mean: $578.1 \mathrm{pmol} / \mathrm{mg}$; range: 243.7-1136.2 $\mathrm{pmol} / \mathrm{mg}$ ). This is caused by the fact that the OMNIgene-GUT-collected samples are diluted in stabilizer (approximately $500 \mathrm{mg}$ of sample in $1.5 \mathrm{ml}$ stabilizing liquid), resulting in lower total fecal BA concentrations.

Variation between each donors' BA profile is detectable using the two sample collection methods (e.g. the BA profiles of Donors 1 and 2, which are samples collected from children under the age of 2, are readily differentiated among the rest of the donors) (Fig. 3).

No significant difference was found in the relative concetrations of primary unconjugated BAs (CA, CDCA) to the total BA concentrations in OMNIgene-GUT-collected samples as compared with the snap frozen samples (Wilcoxon-Mann-Whitney test, $\mathrm{p}>0.05$ ) (Table 1 and Fig. 3). The same result was observed with the relative concentrations of secondary unconjugated BAs (DCA, LCA, UDCA) to the total BA concentrations in both sample collection methods.

To evaluate the degree of correlation in relative concentrations of bile acids between OMNIgene-GUT vs snap frozen samples, we performed Passing-Bablok method comparison and correlation analysis for bile acids with average relative concentration higher than 0.01 . Passing-Bablok regression showed a high degree of correlation in the relative concentrations of CA, CDCA, DCA and LCA between OMNIgene-GUT and snap frozen samples (Fig. 4). For these four bile acids, the two methods are comparable at an acceptability bias of $30 \%$. 


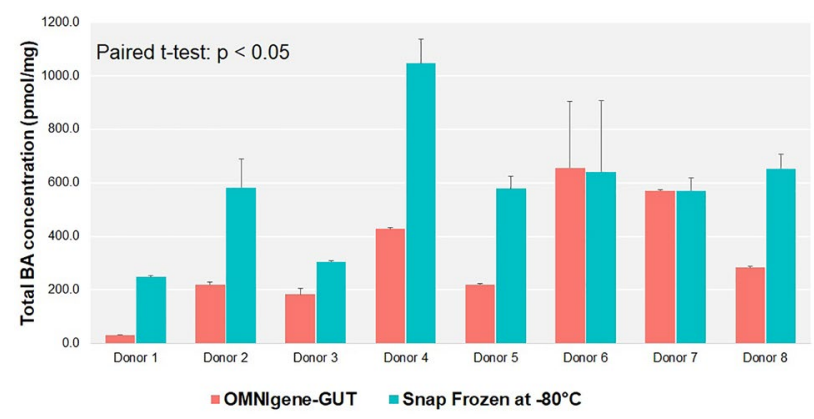

Figure 2. Quantification of endogenous bile acids, measured from eight human stool samples of different mass and stabilized in OMNIgene-GUT and Snap frozen at $-80^{\circ} \mathrm{C}$. Results are shown in terms of total fecal bile acid concentrations (pmol) per mg stool. Each bar represents the mean value of duplicate measurements per donor. The error bars represent one standard deviation. Paired t-test, $\mathrm{p}=0.0029$.

\section{Discussion}

Excellent reviews of preanalytical aspects of fecal metabolomics have been published ${ }^{11,12}$, highlighting the variability in the protocols used for fecal sample collection, processing (metabolite extraction) and metabolite analysis, the impact of such variations on the recovery of fecal metabolites, and the need for both preanalytical and analytical guidelines. It has specifically been shown that the type of solvent used, as well as the ratio fecal:solvent influence the accuracy of analyses by nuclear magnetic resonance (NMR), while freeze thaw and sonication have less impact ${ }^{13,14}$. In this study, we used a fully validated analytical method [Pham et. al, 2016] that has been optimized by Biocrates for use with plasma samples ${ }^{15}$. This validated protocol was adapted for stool samples using a modified protocol published by the same manufacturer ${ }^{16}$.A study by De Spiegeleer et al. using lyophilized fecal samples, has shown that freeze thawing affects the polar more than the lipid metabolome, with $10 \%$ and $7 \%$ of metabolites showing statistically significant differences after one freeze thaw cycle ${ }^{17}$. Previous studies have also shown an impact of freeze thawing, probably due to reactivation of microbial biochemical reactions upon thawing, however such changes were small relative to inter-individual differences ${ }^{18}$. In our study, all samples underwent two freeze thaw cycles before analysis, and it can be assumed that the potential bias was the same in all samples.

RNAlater is a very commonly used stabiliser for nucleic acids in different types of biospecimens, including feces. However, it has been repeatedly shown that RNAlater is not fit for purpose for any metabolomics analyses, probably due to high sodium sulphate content ${ }^{7,8,19}$. There has been a previous preanalytical study, including OMNIgene-GUT tubes, and comparing them to snap frozen fecal samples, and samples collected in ethanol, RNAlater or on FTA cards, in the scope of 16S rRNA gene sequencing, and untargeted and targeted metabolomics ${ }^{7}$. This study showed that OMNIgene-GUT stabiliser is suboptimal for untargeted metabolomics analyses, due to lower sensitivity, but fit for purpose for targeted metabolomics on short chain fatty acids (SCFA), with high concordance with the snap frozen samples. A more recent study by Lim et. al. suggested that it is possible to extract both microbiome and metabolite data from a single stool sample collected using OMNIgene-GUT tubes $^{20}$. Our study has also found fitness for purpose of OMNIgene-GUT tubes for targeted metabolomics for another class of metabolites, the bile acids.

We conclude that the OMNIgene-GUT-collected stool samples are fit-for-purpose for downstream metabolomics assays in the scope of quantitative fecal bile acids analysis. However, the reference ranges of the total and individual fecal BA concentrations are different in OMNIgene-GUT-collected samples and in snap frozen samples. Therefore, the values obtained from the two collection methods cannot be compared directly. However, there is a high degree of correlation in the relative concentrations of CA, CDCA, DCA and LCA between OMNIgene-GUT and snap frozen samples.

Finally, at the time of writing of this article, a new stool collection device has been commercialized by DNA Genotek, the OMNImet-GUT stool collection device, which is declared by the manufacturer to be specifically fit for purpose for metabolomics analyses.

\section{Methods}

Sample collection. For the comparison of snap frozen and OMNIgene-GUT-collected stool samples in the scope of fecal BA quantification, fresh stool samples were collected by 8 healthy donors in a sterile stool collection container (Sarstedt, ref: 80.734.311) and brought to the Integrated BioBank of Luxembourg (IBBL) laboratory within 2-3 h of collection. This study was approved by the National Research Ethics Committee of Luxembourg (Comité National d'Ethique de Recherche-CNER approval \#201,107/02). All experiments and analyses were executed in accordance with the approved guidelines and relevant regulations, and informed consent was obtained from each participant.

Upon arrival in the laboratory, each fresh donor sample was then aliquoted into two separate containers: the OMNIgene-GUT kit (DNA Genotek, ref: OMR-200) and plain $2 \mathrm{ml}$ cryovial (Greiner, ref: 126,263-2D1). For the OMNIgene-GUT kit, approximately $500 \mathrm{mg}$ of fresh sample was placed in the tube containing the stabilizer. The tube was homogenized by mixing and stored temporarily at room temperature (RT) for 3-5 days to mimic RT 


\begin{tabular}{|c|c|c|c|c|c|c|c|c|c|c|c|c|c|c|c|c|c|}
\hline \multirow{2}{*}{$\begin{array}{l}\text { Donor } \\
\mathbf{N}^{\circ}\end{array}$} & \multirow{2}{*}{$\begin{array}{l}\text { Storage } \\
\text { container }\end{array}$} & \multirow[b]{2}{*}{ Sample ID } & \multicolumn{15}{|c|}{ Bile acid concentration (pmol/mg) } \\
\hline & & & CA & CDCA & DCA & LCA & UDCA & GCA & GCDCA & GDCA & GLCA & GUDCA & TCA & TCDCA & TDCA & TLCA & TUDCA \\
\hline \multirow{4}{*}{$\begin{array}{l}\text { Donor } \\
1\end{array}$} & \multirow{2}{*}{$\begin{array}{l}\text { OMNI- } \\
\text { gene-GUT }\end{array}$} & $\begin{array}{l}\text { D1-OMNIg- } \\
\text { GUT_1 }\end{array}$ & 22.92 & 32.236 & 23.788 & 0.6908 & 0 & 0.0648 & 0.478 & 0 & 0 & 0 & 0.0408 & 0.0816 & 0 & 0 & 0.0012 \\
\hline & & $\begin{array}{l}\text { D1-OMNIg- } \\
\text { GUT_2 }\end{array}$ & 24.12 & 31.784 & 2.454 & 0.9 & 0 & 0.0476 & 0.4244 & 0 & 0 & 0 & 0.0284 & 0.0832 & 0 & 0 & 0.002 \\
\hline & \multirow{2}{*}{$\begin{array}{l}\text { Snap } \\
\text { frozen at } \\
-80^{\circ} \mathrm{C}\end{array}$} & $\begin{array}{l}\text { D1-SnapFro- } \\
\text { zen_1 }\end{array}$ & 206 & 21.2 & 15.2 & 0.8184 & 0 & 0.0876 & 0.0708 & 0 & 0 & 0 & 0.246 & 0.036 & 0.0168 & 0 & 0 \\
\hline & & $\begin{array}{l}\text { D1-SnapFro- } \\
\text { zen_2 }\end{array}$ & 213.6 & 22.12 & 15.84 & 0.6676 & 0 & 0.1388 & 0.0748 & 0 & 0 & 0 & 0.238 & 0.0376 & 0.0244 & 0 & 0 \\
\hline \multirow{4}{*}{$\begin{array}{l}\text { Donor } \\
2\end{array}$} & \multirow{2}{*}{$\begin{array}{l}\text { OMNI- } \\
\text { gene-GUT }\end{array}$} & \begin{tabular}{|l}
$\begin{array}{l}\text { D2-OMNIg- } \\
\text { GUT_1 }\end{array}$ \\
\end{tabular} & 152.4 & 29 & 20.56 & 0.4924 & 1.652 & 19.776 & 27.952 & 0 & 0 & 0.0532 & 0.4396 & 0.6764 & 0 & 0 & 0.0156 \\
\hline & & $\begin{array}{l}\text { D2-OMNIg- } \\
\text { GUT_2 }\end{array}$ & 166.8 & 32 & 22.6 & 0.6576 & 1.794 & 22.348 & 29.308 & 0 & 0 & 0.0716 & 0.4568 & 0.7972 & 0 & 0 & 0.0148 \\
\hline & \multirow{2}{*}{$\begin{array}{l}\text { Snap } \\
\text { frozen at } \\
-80^{\circ} \mathrm{C}\end{array}$} & $\begin{array}{l}\text { D2-SnapFro- } \\
\text { zen_1 }\end{array}$ & 385.6 & 48 & 33.32 & 13.252 & 36.196 & 14.224 & 0.9752 & 0 & 0 & 0.0376 & 0.446 & 0.448 & 0 & 0 & 0.034 \\
\hline & & $\begin{array}{l}\text { D2-SnapFro- } \\
\text { zen_2 }\end{array}$ & 548 & 78 & 52.4 & 0.5364 & 5.16 & 20.348 & 13.344 & 0 & 0 & 0.0544 & 0.3296 & 0.4364 & 0 & 0 & 0.044 \\
\hline \multirow{4}{*}{$\begin{array}{l}\text { Donor } \\
3\end{array}$} & \multirow{2}{*}{$\begin{array}{l}\text { OMNI- } \\
\text { gene-GUT }\end{array}$} & $\begin{array}{l}\text { D3-OMNIg- } \\
\text { GUT_1 }\end{array}$ & 22.076 & 0.1596 & 110.4 & 86 & 0 & 10.688 & 18.092 & 11.412 & 0 & 0.106 & 18.236 & 0.9068 & 0.3808 & 0 & 0.062 \\
\hline & & $\begin{array}{l}\text { D3-OMNIg- } \\
\text { GUT_2 }\end{array}$ & 16.552 & 0.1332 & 91.2 & 61.6 & 0 & 0.9096 & 10.468 & 0.4844 & 0 & 0.08 & 11.816 & 0.6268 & 0.256 & 0 & 0.0372 \\
\hline & \multirow{2}{*}{$\begin{array}{l}\text { Snap } \\
\text { frozen at } \\
-80^{\circ} \mathrm{C}\end{array}$} & $\begin{array}{l}\text { D3-SnapFro- } \\
\text { zen_1 }\end{array}$ & 31.556 & 0.03 & 98.4 & 204 & 12.944 & 0.0844 & 0.5056 & 0.5636 & 0.2836 & 0.0496 & 0.1656 & 0.3156 & 0.3196 & 0.156 & 0.046 \\
\hline & & $\begin{array}{l}\text { D3-SnapFro- } \\
\text { zen_2 }\end{array}$ & 3.552 & 0 & 90.4 & 204 & 10.176 & 0.128 & 0.6068 & 0.6392 & 0.2492 & 0.0984 & 0.5336 & 0.5396 & 0.382 & 0.108 & 0.048 \\
\hline \multirow{4}{*}{$\begin{array}{l}\text { Donor } \\
4\end{array}$} & \multirow{2}{*}{$\begin{array}{l}\text { OMNI- } \\
\text { gene-GUT }\end{array}$} & $\begin{array}{l}\text { D4-OMNIg- } \\
\text { GUT_1 }\end{array}$ & 0.8408 & 10.116 & 278.8 & 148.8 & 0 & 0.1452 & 0.7988 & 16.992 & 0 & 0.062 & 0.0404 & 0.0628 & 0.09 & 0 & 0 \\
\hline & & $\begin{array}{l}\text { D4-OMNIg- } \\
\text { GUT_2 }\end{array}$ & 0.9276 & 1.036 & 265.2 & 152 & 0 & 0.1388 & 0.8672 & 17.252 & 0 & 0.0356 & 0.0444 & 0.0592 & 0.082 & 0 & 0 \\
\hline & \multirow{2}{*}{$\begin{array}{l}\text { Snap } \\
\text { frozen at } \\
-80^{\circ} \mathrm{C}\end{array}$} & $\begin{array}{l}\text { D4-SnapFro- } \\
\text { zen_1 }\end{array}$ & 3.15 & 7.64 & 752 & 365.2 & 7.2 & 0.0584 & 0.1004 & 0.5824 & 0.194 & 0.0344 & 0 & 0.0084 & 0.0588 & 0 & 0 \\
\hline & & $\begin{array}{l}\text { D4-SnapFro- } \\
\text { zen_2 }\end{array}$ & 14.476 & 2.074 & 596 & 351.2 & 39.876 & 0.0696 & 0.1032 & 0.6424 & 0.1152 & 0.0508 & 0 & 0.0056 & 0.042 & 0 & 0 \\
\hline \multirow{4}{*}{$\begin{array}{l}\text { Donor } \\
5\end{array}$} & \multirow{2}{*}{$\begin{array}{l}\text { OMNI- } \\
\text { gene-GUT }\end{array}$} & $\begin{array}{l}\text { D5-OMNIg- } \\
\text { GUT_1 }\end{array}$ & 0.9956 & 1.134 & 83.2 & 134 & 0 & 0.8676 & 1.816 & 0.9612 & 0 & 0.0564 & 0.1436 & 0.2168 & 0.114 & 0.0124 & 0.006 \\
\hline & & $\begin{array}{l}\text { D5-OMNIg- } \\
\text { GUT_2 }\end{array}$ & 0.9584 & 10.256 & 81.2 & 128 & 0 & 0.7504 & 14.456 & 0.9028 & 0 & 0.056 & 0.1408 & 0.2056 & 0.1 & 0.0144 & 0.0064 \\
\hline & \multirow{2}{*}{$\begin{array}{l}\text { Snap } \\
\text { frozen at } \\
-80^{\circ} \mathrm{C}\end{array}$} & $\begin{array}{l}\text { D5-SnapFro- } \\
\text { zen_1 }\end{array}$ & 10.684 & 32.744 & 190.8 & 332 & 0 & 0.1704 & 0.4692 & 0.5532 & 0.1468 & 0.0344 & 0.1496 & 0.2536 & 0.2588 & 0.0632 & 0.0084 \\
\hline & & $\begin{array}{l}\text { D5-SnapFro- } \\
\text { zen_2 }\end{array}$ & 20.968 & 0.9776 & 236 & 383.6 & 0 & 0.3192 & 0.4056 & 0.6488 & 0.2052 & 0.1336 & 0.662 & 0.2068 & 0.3836 & 0.06 & 0 \\
\hline \multirow{4}{*}{$\begin{array}{l}\text { Donor } \\
6\end{array}$} & \multirow{2}{*}{$\begin{array}{l}\text { OMNI- } \\
\text { gene-GUT }\end{array}$} & $\begin{array}{l}\text { D6-OMNIg- } \\
\text { GUT_1 }\end{array}$ & 0.1608 & 0.2136 & 206.4 & 200.8 & 0 & 0.2296 & 0.7376 & 0.7228 & 0 & 0.046 & 0.0616 & 0.0884 & 0.0884 & 0.026 & 0 \\
\hline & & $\begin{array}{l}\text { D6-OMNIg- } \\
\text { GUT_2 }\end{array}$ & 0.7508 & 1.364 & 460 & 440 & 0 & 0.1016 & 0.124 & 0.5484 & 0 & 0.0924 & 0.2632 & 0.0968 & 0.2508 & 0.1104 & 0.0432 \\
\hline & \multirow{2}{*}{$\begin{array}{l}\text { Snap } \\
\text { frozen at } \\
-80^{\circ} \mathrm{C}\end{array}$} & $\begin{array}{l}\text { D6-SnapFro- } \\
\text { zen_1 }\end{array}$ & 0.7364 & 9.76 & 468 & 428 & 0 & 0.1264 & 0.1384 & 0.4332 & 0 & 0.1148 & 0.1656 & 0.0584 & 0.1948 & 0.1424 & 0 \\
\hline & & $\begin{array}{l}\text { D6-SnapFro- } \\
\text { zen_2 }\end{array}$ & 0.7612 & 0.2624 & 178.8 & 193.6 & 0 & 0.2248 & 0.6368 & 0.6672 & 0 & 0.0476 & 0.0348 & 0.0776 & 0.0764 & 0.0152 & 0 \\
\hline \multirow{4}{*}{$\begin{array}{l}\text { Donor } \\
7\end{array}$} & OMNI- & $\begin{array}{l}\text { D7-OMNIg- } \\
\text { GUT_1 }\end{array}$ & 29.356 & 0.5664 & 408 & 157.2 & 0 & 0.7192 & 0.6348 & 37.236 & 0 & 0 & 0.1648 & 0.0604 & 0.2912 & 0 & 0 \\
\hline & gene-GUT & $\begin{array}{l}\text { D7-OMNIg- } \\
\text { GUT_2 }\end{array}$ & 29.956 & 0.53 & 408 & 150.4 & 0 & 0.7264 & 0.6436 & 3.848 & 0 & 0.0328 & 0.1876 & 0.066 & 0.3076 & 0 & 0.0116 \\
\hline & & $\begin{array}{l}\text { D7-SnapFro- } \\
\text { zen_1 }\end{array}$ & 12.044 & 0 & 408 & 209.2 & 0 & 0.096 & 0.044 & 0.7248 & 0.2196 & 0 & 0 & 0.014 & 0.1508 & 0.042 & 0.0196 \\
\hline & $\begin{array}{l}\text { Irozen at } \\
-80^{\circ} \mathrm{C}\end{array}$ & $\begin{array}{l}\text { D7-SnapFro- } \\
\text { zen_2 }\end{array}$ & 0.9796 & 0 & 331.2 & 188.4 & 0 & 0.1696 & 0.0948 & 0.5992 & 0.1488 & 0 & 0.0332 & 0.0368 & 0.17 & 0.0516 & 0 \\
\hline & OMNI- & $\begin{array}{l}\text { D8-OMNIg- } \\
\text { GUT_1 }\end{array}$ & 10.076 & 2.018 & 134 & 135.2 & 0 & 11.496 & 1.402 & 0.796 & 0 & 0.1288 & 0.2836 & 0.3304 & 0.1188 & 0 & 0.0192 \\
\hline Donor & gene-GUT & $\begin{array}{l}\text { D8-OMNIg- } \\
\text { GUT_2 }\end{array}$ & 1.174 & 2.184 & 143.2 & 136.4 & 0.576 & 12.972 & 17.156 & 1.036 & 0 & 0.1432 & 0.3096 & 0.4008 & 0.1412 & 0 & 0.0168 \\
\hline & Snap & $\begin{array}{l}\text { D8-SnapFro- } \\
\text { zen_1 }\end{array}$ & 14.484 & 0.5604 & 338 & 364 & 0 & 0.2664 & 0.7544 & 0.5436 & 0 & 0.136 & 0.0936 & 0.2856 & 0.1176 & 0 & 0.0108 \\
\hline & $\begin{array}{l}\text { frozen at } \\
-80^{\circ} \mathrm{C}\end{array}$ & $\begin{array}{l}\text { D8-SnapFro- } \\
\text { zen_2 }\end{array}$ & 26.372 & 3.206 & 273.6 & 317.6 & 15.684 & 0.3276 & 0.4696 & 0.272 & 0 & 0.0908 & 0.0592 & 0.12 & 0.0724 & 0 & 0 \\
\hline
\end{tabular}

Table 1. Bile Acid Concentration (pmol/mg) of each sample tested per donor. CA-Cholic Acid; CDCAChenodeoxycholic Acid; DCA-Deoxycholic Acid; LCA-Lithocholic Acid; UDCA-Ursodeoxycholic Acid; GCA-Glycocholic Acid; GCDCA- Glycochenodeoxycholic Acid; GDCA-Glycodeoxycholic Acid; GLCA-Glycolithocholic Acid; GUDCA-Glycoursodeoxycholic Acid; TCA-Taurocholic Acid; TCDCATaurochenodeoxycholic Acid; TDCA-Taurodeoxycholic Acid; TLCA-Taurolithocholic Acid; TUDCATauroursodeoxycholic Acid. 


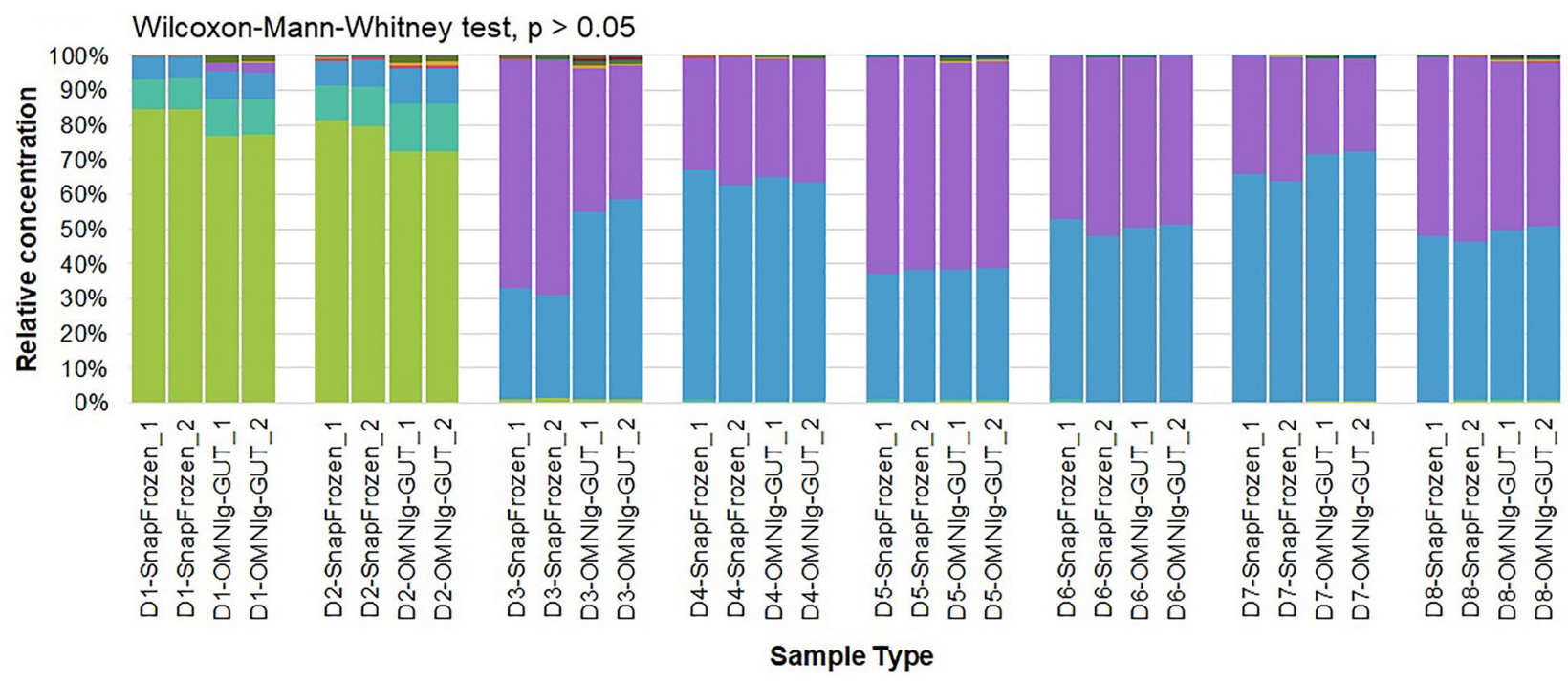

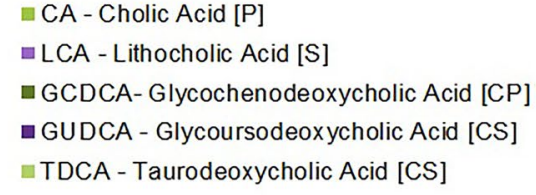

\author{
a CDCA - Chenodeoxycholic Acid [P] \\ -UDCA - Ursodeoxycholic Acid [S] \\ - GDCA - Glycodeoxycholic Acid [CS] \\ -TCA - Taurocholic Acid [CP] \\ $\square$ TLCA - Taurolithocholic Acid [CS]
}

\author{
- DCA - Deoxycholic Acid [S] \\ $\square$ GCA - Glycocholic Acid [CP] \\ - GLCA - Glycolithocholic Acid [CS] \\ $\because$ TCDCA - Taurochenodeoxycholic Acid [CP] \\ -TUDCA - Tauroursodeoxycholic Acid [CS]
}

Figure 3. Visual comparison of the relative concentrations of individual bile acids for each sample type (OMNIgene-GUT and Snap frozen at $-80^{\circ} \mathrm{C}$ ) measured in eight donor samples, based on the quantification of bile acids by LC-MS/MS analysis method. (Wilcoxon-Mann-Whitney test, $\mathrm{p}>0.05$ ). $[\mathrm{P}]=$ primary bile acid; $[\mathrm{S}]=$ secondary bile acid; $[\mathrm{CP}]=$ conjugated primary bile acid; $[\mathrm{CS}]=$ conjugated secondary bile acid.

transport conditions. After storage at RT, the sample in the OMNIgene-GUT tube was aliquoted into two 2-ml cryovial containing $1 \mathrm{ml}$ of stool suspension each and frozen at $-80{ }^{\circ} \mathrm{C}$ for two weeks.

For the snap frozen sample, approximately $1 \mathrm{~g}$ of the fresh donor sample was placed in a plain $2 \mathrm{ml}$ cryovial. This cryovial was frozen immediately in $-80^{\circ} \mathrm{C}$ for two weeks.

Sample preparation. Eight OMNIgene-GUT-stabilized samples and eight snap frozen samples were thawed and each sample was further aliquoted into another cryovial. The weight of each sample was recorded prior to re-freezing. A total of 32 frozen stool samples were sent to Biocrates Life Sciences AG(Innsbruck, Austria) for the quantification of endogenous bile acids. See Table 2 for sample details.

To extract metabolites from feces, threefold volume of ethanol/phosphate buffer $(85: 15 \mathrm{v} / \mathrm{v})$ was added to each stool sample and was vortexed for $3 \mathrm{~min}$. The homogenized samples were then placed in a plate shaker for $30 \mathrm{~min}$ at $200 \mathrm{rpm}$ at $0{ }^{\circ} \mathrm{C}$. Following this step, the samples were sonicated at $70 \mathrm{~W}$ for $5 \mathrm{~min}$ at $0{ }^{\circ} \mathrm{C}$ and centrifuged at $800 \mathrm{~g}$ for $10 \mathrm{~min}$ at $0^{\circ} \mathrm{C}$. The supernatant from each tube was then transferred into a new reaction tube and centrifuged at $19,000 \mathrm{~g}$ for $10 \mathrm{~min}$ at $4^{\circ} \mathrm{C}$. The new supernatants from this step were transferred into new reaction tubes and were used for analysis. $10 \mu \mathrm{L}$ of supernatant/fecal extracts were used for the Biocrates' Bile Acids Kit according to the manufacturers' instructions.

Quantification of fecal BA. The endogenous bile acids were quantified in collaboration with the Metabolic Phenotyping Services Center of Biocrates Life Sciences AG, (Innsbruck, Austria) using a mass spectrometric-based metabolomic approach with the commercially-available Biocrates' Bile Acids Kit ${ }^{21}$. A highly selective reversed phase LC-MS/MS analysis method in negative ion multiple reaction monitoring (MRM) detection mode was applied to determine the concentrations of bile acids. The samples were extracted via dried filter spot technique in 96-well plate format. Sample extracts were measured by LC-ESI-MS/MS with a tandem mass spectrometry instrument (TSQ Vantage, Thermo Fisher Scientific TSQ). For highly accurate quantification, 7-point external calibration curves and 10 stable isotope-labeled internal standards were applied. Data of bile acids were quantified using the appropriate MS software (Thermo Fisher Scientific Xcalibur) and the results were finally imported into Biocrates MetIDQ software for further analysis.

Accuracy of the measurements (determined with the accuracy of the calibrators) was in the normal range of the method (deviations from target $\leq 20 \%$ ) for all analytes. Quality control samples were within the pre-defined tolerances of the method. 
A

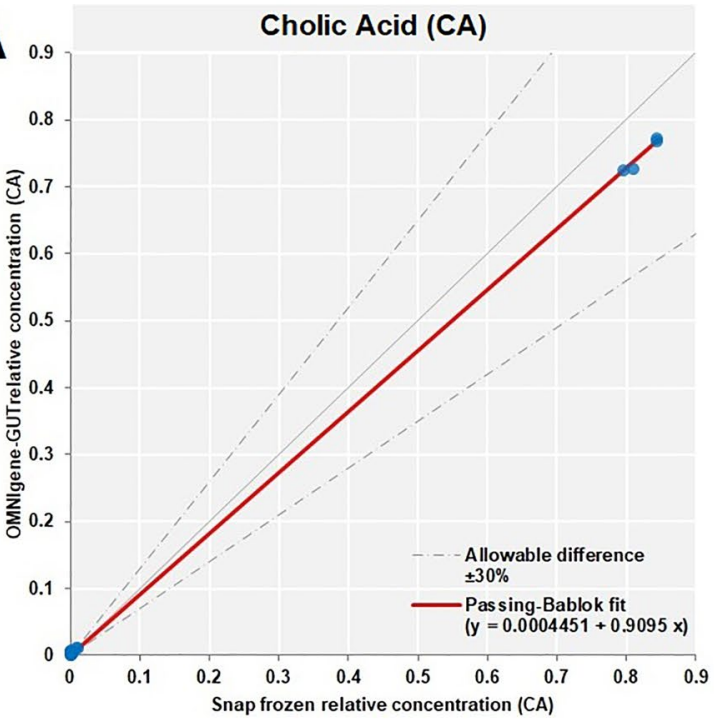

C

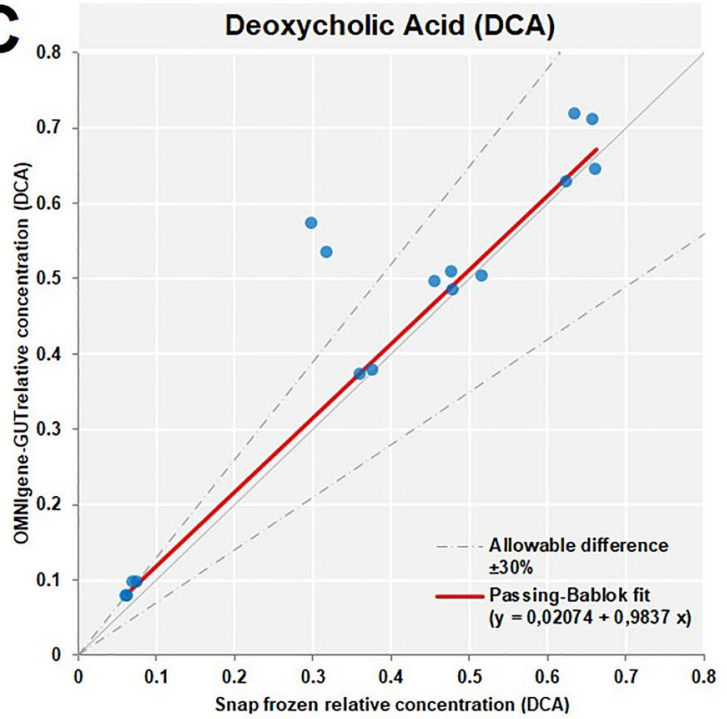

B

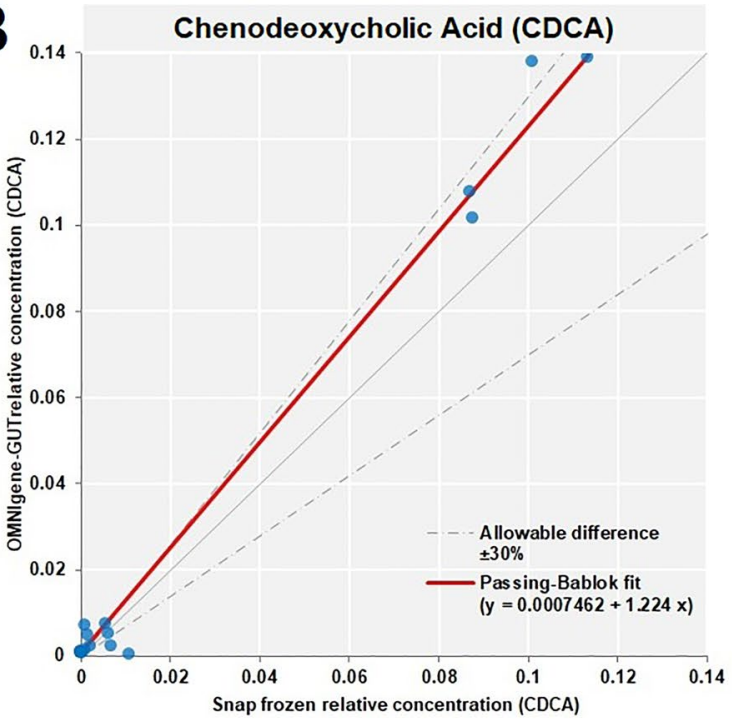

D

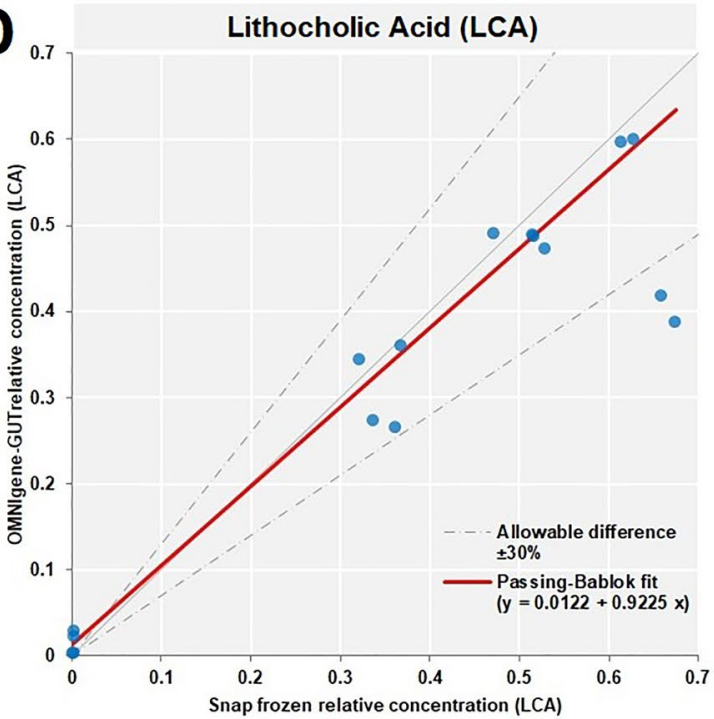

Figure 4. Passing-Bablok method comparison and correlation analysis for bile acids with average relative concentration higher than 0.01. The relative concentrations of Cholic Acid (A), Chenodeoxycholic Acid (B), Deoxycholic Acid (C) and Lithocholic Acid (D) in OMNIgene-GUT and snap frozen samples are plotted and indicated by the blue points. The snap frozen is taken as the reference method, while the OMNIgene-GUT is taken as the test method. The Passing-Bablok fit is shown as the red line. The allowable bias between the relative concentrations in OMNIgene-GUT and snap frozen samples is $30 \%$.

Statistical analyses. All statistical analyses were conducted with Analyse-IT Method Validation Edition, v.4.60.01 (Analyse-it Software, Ltd.) and Microsoft Office Excel 2016. The analysis of distribution of total BAs data was done by performing Shapiro-Wilk test. Paired t-test was performed to compare the total BAs in both OMNIgene-GUT and snap frozen samples. The comparison between the relative concentrations of primary BAs (CA, CDCA) and secondary BAs (DCA, LCA, UDCA) to the total BA concentrations in OMNIgene-GUT-collected samples and snap frozen samples were conducted using the Wilcoxon-Mann-Whitney test. For bile acids with an average relative concentration higher than 0.01 (CA, CDCA, DCA and LCA), Passing-Bablok method comparison and correlation analysis was performed. For all the tests performed, the statistical significance was accepted at $\mathrm{p}<0.05$. 


\begin{tabular}{|c|c|c|c|}
\hline Donor $\mathbf{N}^{\circ}$ & Storage Container & Sample ID & Amount used for fecal BA quantification (weight in grams) \\
\hline \multirow{4}{*}{ Donor 1} & \multirow{2}{*}{ OMNIgene-GUT } & D1-OMNIg-GUT_1 & 0.6503 \\
\hline & & D1-OMNIg-GUT_2 & 0.2906 \\
\hline & \multirow{2}{*}{ Snap frozen at $-80^{\circ} \mathrm{C}$} & D1-SnapFrozen_1 & 0.1993 \\
\hline & & D1-SnapFrozen_2 & 0.1452 \\
\hline \multirow{4}{*}{ Donor 2} & \multirow{2}{*}{ OMNIgene-GUT } & D2-OMNIg-GUT_1 & 0.6685 \\
\hline & & D2-OMNIg-GUT_2 & 0.5691 \\
\hline & \multirow{2}{*}{ Snap frozen at $-80^{\circ} \mathrm{C}$} & D2-SnapFrozen_1 & 0.5736 \\
\hline & & D2-SnapFrozen_2 & 0.2074 \\
\hline \multirow{4}{*}{ Donor 3} & \multirow{2}{*}{ OMNIgene-GUT } & D3-OMNIg-GUT_1 & 0.6368 \\
\hline & & D3-OMNIg-GUT_2 & 0.5140 \\
\hline & \multirow{2}{*}{ Snap frozen at $-80^{\circ} \mathrm{C}$} & D3-SnapFrozen_1 & 0.2304 \\
\hline & & D3-SnapFrozen_2 & 0.1988 \\
\hline \multirow{4}{*}{ Donor 4} & \multirow{2}{*}{ OMNIgene-GUT } & D4-OMNIg-GUT_1 & 0.8189 \\
\hline & & D4-OMNIg-GUT_2 & 0.3327 \\
\hline & \multirow{2}{*}{ Snap frozen at $-80^{\circ} \mathrm{C}$} & D4-SnapFrozen_1 & 0.1878 \\
\hline & & D4-SnapFrozen_2 & 0.1931 \\
\hline \multirow{4}{*}{ Donor 5} & \multirow{2}{*}{ OMNIgene-GUT } & D5-OMNIg-GUT_1 & 0.7210 \\
\hline & & D5-OMNIg-GUT_2 & 0.4504 \\
\hline & \multirow{2}{*}{ Snap frozen at $-80^{\circ} \mathrm{C}$} & D5-SnapFrozen_1 & 0.1989 \\
\hline & & D5-SnapFrozen_2 & 0.1560 \\
\hline \multirow{4}{*}{ Donor 6} & \multirow{2}{*}{ OMNIgene-GUT } & D6-OMNIg-GUT_1 & 0.8290 \\
\hline & & D6-OMNIg-GUT_2 & 0.3123 \\
\hline & \multirow{2}{*}{ Snap frozen at $-80^{\circ} \mathrm{C}$} & D6-SnapFrozen_1 & 0.3690 \\
\hline & & D6-SnapFrozen_2 & 0.2228 \\
\hline \multirow{4}{*}{ Donor 7} & \multirow{2}{*}{ OMNIgene-GUT } & D7-OMNIg-GUT_1 & 0.8288 \\
\hline & & D7-OMNIg-GUT_2 & 0.3430 \\
\hline & \multirow{2}{*}{ Snap frozen at $-80^{\circ} \mathrm{C}$} & D7-SnapFrozen_1 & 0.5330 \\
\hline & & D7-SnapFrozen_2 & 0.2908 \\
\hline \multirow{4}{*}{ Donor 8} & \multirow{2}{*}{ OMNIgene-GUT } & D8-OMNIg-GUT_1 & 0.7669 \\
\hline & & D8-OMNIg-GUT_2 & 0.4655 \\
\hline & \multirow{2}{*}{ Snap frozen at $-80^{\circ} \mathrm{C}$} & D8-SnapFrozen_1 & 0.2328 \\
\hline & & D8-SnapFrozen_2 & 0.1822 \\
\hline
\end{tabular}

Table 2. Details of 32 samples used for the quantification of endogenous bile acids.

Received: 18 August 2020; Accepted: 18 March 2021

Published online: 12 April 2021

\section{References}

1. Begley, M., Gahan, C. G. \& Hill, C. The interaction between bacteria and bile. FEMS Microbiol. Rev. 29(4), 625-651. https://doi. org/10.1016/j.femsre.2004.09.003 (2005).

2. Islam, K. S. et al. Bile acid is a host factor that regulates the composition of the cecal microbiota in rats. Gastroenterology 141(5), 1773-1781. https://doi.org/10.1053/j.gastro.2011.07.046 (2011).

3. Ridlon, J. M., Alves, J. M., Hylemon, P. B. \& Bajaj, J. S. Cirrhosis, bile acids and gut microbiota. Gut Microbes 4(5), 382-387. https:// doi.org/10.4161/gmic.25723 (2013).

4. Hylemon, P. B. et al. Bile acids as regulatory molecules. J. Lipid Res. 50(8), 1509-1520. https://doi.org/10.1194/jlr.r900007-jlr200 (2009).

5. Hofmann, A. F. Bile acids: Trying to understand their chemistry and biology with the hope of helping patients. Hepatology 49(5), 1403-1418. https://doi.org/10.1002/hep.22789 (2008).

6. Mouzaki, M. et al. Bile acids and dysbiosis in non-alcoholic fatty liver disease. PLoS ONE 11(5), 1. https://doi.org/10.1371/journ al.pone.0151829(2016).

7. Wang, Z. et al. Comparison of fecal collection methods for microbiome and metabolomics studies. Front. Cell. Infect. Microbiol. https://doi.org/10.3389/fcimb.2018.00301 (2018).

8. Loftfield, E. et al. Comparison of collection methods for fecal samples for discovery metabolomics in epidemiologic studies. Cancer Epidemiol. Biomarkers Prev. 25, 1483-1490. https://doi.org/10.1158/1055-9965.EPI-16-0409 (2016).

9. Neuberger-Castillo, L. et al. Method validation for extraction of DNA from human stool samples for downstream microbiome analysis. Biopreserv. Biobank. 18(2), 102-116. https://doi.org/10.1089/bio.2019.0112 (2020).

10. DNA Genotek (2020). OMNIgene-GUT Product Description. Document No. PD-BR-00181 issue 6/2020-03 https://www.dnage notek.com/ROW/pdf/PD-BR-00181.pdf

11. Deda, O., Gika, H. G., Wilson, I. D. \& Theodoridis, G. A. An overview of fecal sample preparation for global metabolic profiling. J. Pharm. Biomed. Anal. 113, 137-150. https://doi.org/10.1016/j.jpba.2015.02.006 (2015)

12. Matysik, S., Roy, C. I., Liebisch, G. \& Claus, S. P. Metabolomics of fecal samples: A practical consideration. Trends Food Sci. Technol. 57, 244-255. https://doi.org/10.1016/j.tifs.2016.05.011 (2016). 
13. Jacobs, D. M. et al. 1H NMR metabolite profiling of feces as a tool to assess the impact of nutrition on the human microbiome. NMR Biomed. 21(6), 615-626. https://doi.org/10.1002/nbm.1233 (2007).

14. Lamichhane, S. et al. Strategy for nuclear-magnetic-resonance-based metabolomics of human feces. Anal. Chem. 87(12), 59305937. https://doi.org/10.1021/acs.analchem.5b00977 (2015).

15. Pham, H. T. et al. Inter-laboratory robustness of next-generation bile acid study in mice and humans: international ring trial involving 12 laboratories. J. Appl. Lab. Med. AACC Publ. 1(2), 129-142. https://doi.org/10.1373/jalm.2016.020537 (2016).

16. Biocrates (2016). Analysis of Human Fecal Samples with the Biocrates Bile Acids Kit. Application Note No. 35001, V1.0, 23 February 2016.

17. Spiegeleer, M. D. et al. Impact of storage conditions on the human stool metabolome and lipidome: Preserving the most accurate fingerprint. Anal. Chim. Acta 1108, 79-88. https://doi.org/10.1016/j.aca.2020.02.046 (2020).

18. Gratton, J. et al. Optimized Sample Handling Strategy for Metabolic Profiling of Human Feces. Anal. Chem. 88(9), 4661-4668. https://doi.org/10.1021/acs.analchem.5b04159 (2016).

19. Sinha, R. et al. Collecting Fecal Samples for Microbiome Analyses in Epidemiology Studies. Cancer Epidemiol. Biomark. Prev. 25(2), 407-416. https://doi.org/10.1158/1055-9965.epi-15-0951 (2015).

20. Lim, M. Y. et al. Changes in microbiome and metabolomic profiles of fecal samples stored with stabilizing solution at room temperature: a pilot study. Sci. Rep. 10, 1789. https://doi.org/10.1038/s41598-020-58719-8 (2020).

21. Biocrates (2019). Biocrates Bile Acids Kit. Application Note No. 35028, V2.0, February 2019. https://biocrates.com/wp-content/ uploads/2020/02/Biocrates_Bileacids.pdf

\section{Acknowledgments}

The authors would like to thank Biocrates Life Sciences AG (Innsbruck, Austria) for their technical support.

\section{Author contributions}

L.N.C. performed data curation, formal analysis, visualization, original draft preparation, review and editing. W.A. performed formal analysis, review and editing. F.B. performed conceptualization while at IBBL, formal analysis while at LNS, supervision, original draft preparation, review and editing while at LNS. All authors reviewed and approved the manuscript.

\section{Competing interests}

The authors declare no competing interests.

\section{Additional information}

Correspondence and requests for materials should be addressed to L.N.-C.

Reprints and permissions information is available at www.nature.com/reprints.

Publisher's note Springer Nature remains neutral with regard to jurisdictional claims in published maps and institutional affiliations.

Open Access This article is licensed under a Creative Commons Attribution 4.0 International License, which permits use, sharing, adaptation, distribution and reproduction in any medium or format, as long as you give appropriate credit to the original author(s) and the source, provide a link to the Creative Commons licence, and indicate if changes were made. The images or other third party material in this article are included in the article's Creative Commons licence, unless indicated otherwise in a credit line to the material. If material is not included in the article's Creative Commons licence and your intended use is not permitted by statutory regulation or exceeds the permitted use, you will need to obtain permission directly from the copyright holder. To view a copy of this licence, visit http://creativecommons.org/licenses/by/4.0/.

(C) The Author(s) 2021 